\title{
Stable Wearable Strain Sensors on Textiles by Direct Laser Writing of Graphene
}

Wen Liu1,2, Yihe Huang2, Yudong Peng1,2, Monik Walczak3, Dongwang1, Qian Chen1, Zhu Liu1,2* and Lin Li2

1 School of Materials, The University of Manchester, Oxford Road, Manchester M13 9PL, UK

2 Laser Processing Research Centre, School of Mechanical, Aerospace and Civil Engineering, The University of Manchester, Manchester, M13 9PL, UK

3 School of Chemistry, The University of Manchester, Oxford Road, Manchester M13 9PL, UK

*Corresponding author: zhu.liu@manchester.ac.uk 
Diagram of the picosecond laser device

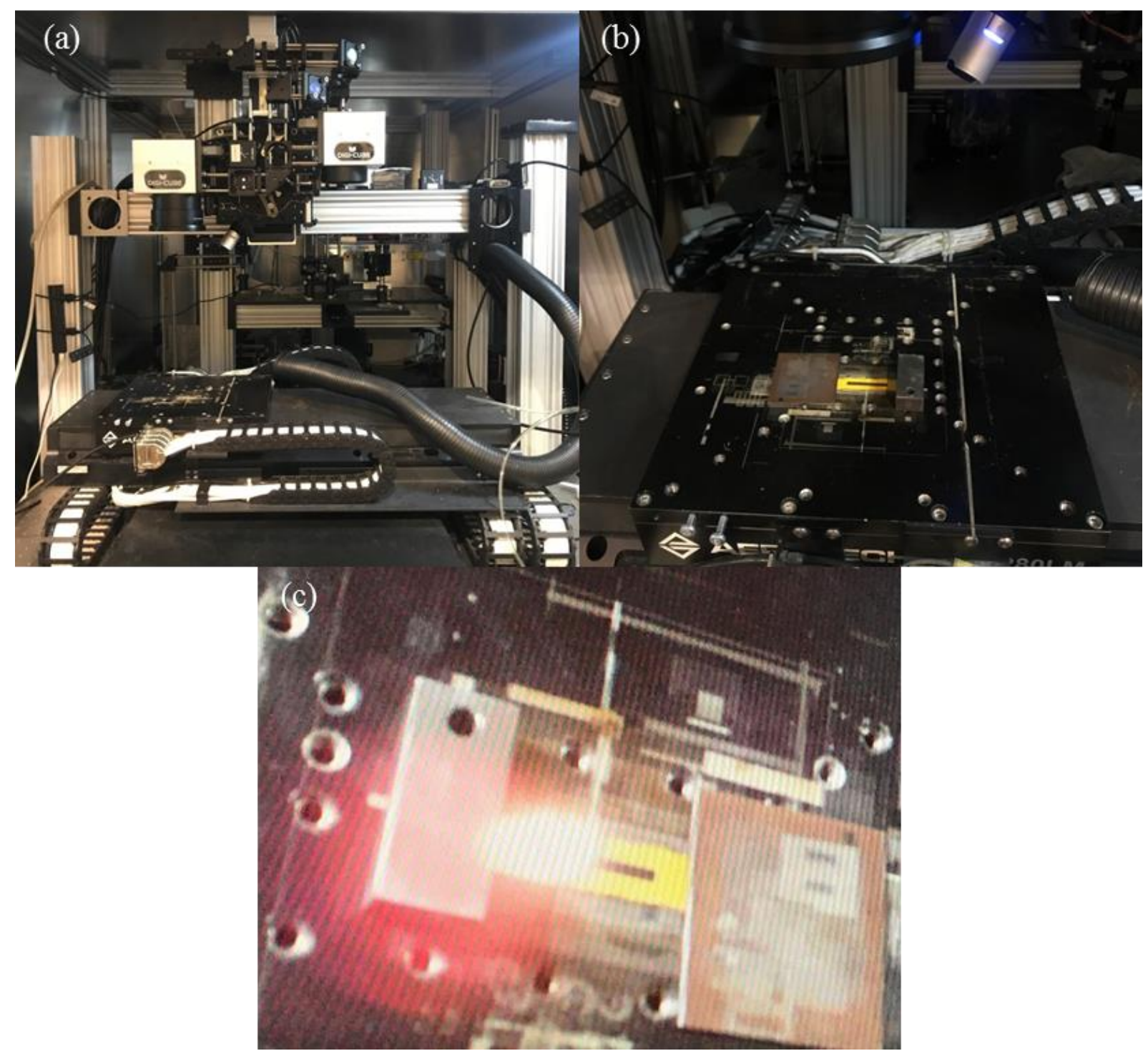

Figure S1. (a) A diagram of the picosecond laser device ;(b) A diagram of the laser processing platform; (c) An image during the process of laser scribing on PI fabric. 


\section{Diagram of mechanical testing machine and resistance recorder}

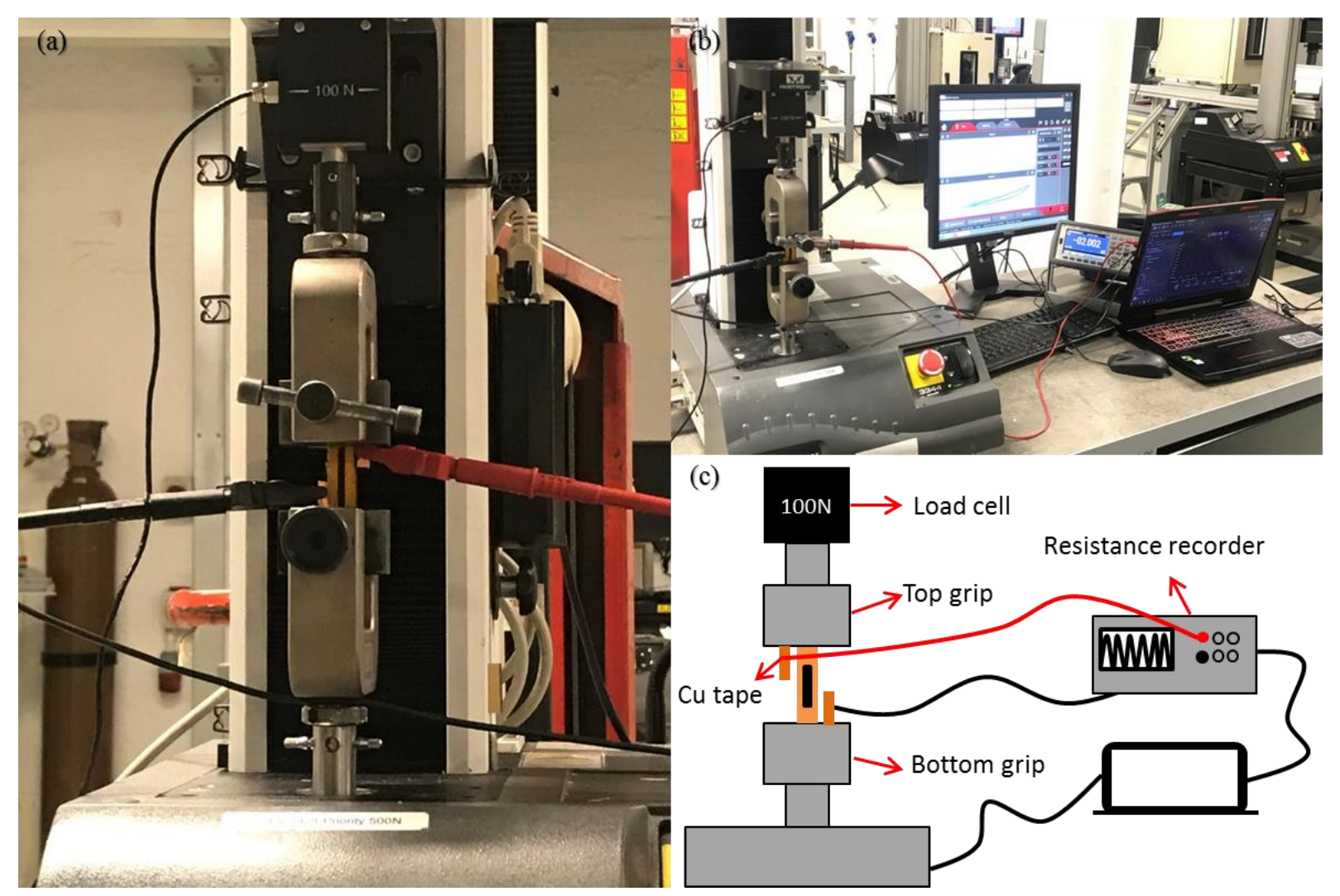

Figure S2. (a) A diagram of Instron 3344L3927 2kN static testing machine;(b) A diagram of electromechanical test setup; (c) An image shows the model structure of electromechanical test setup. 
SEM image of LIG/PI fabric cross-section

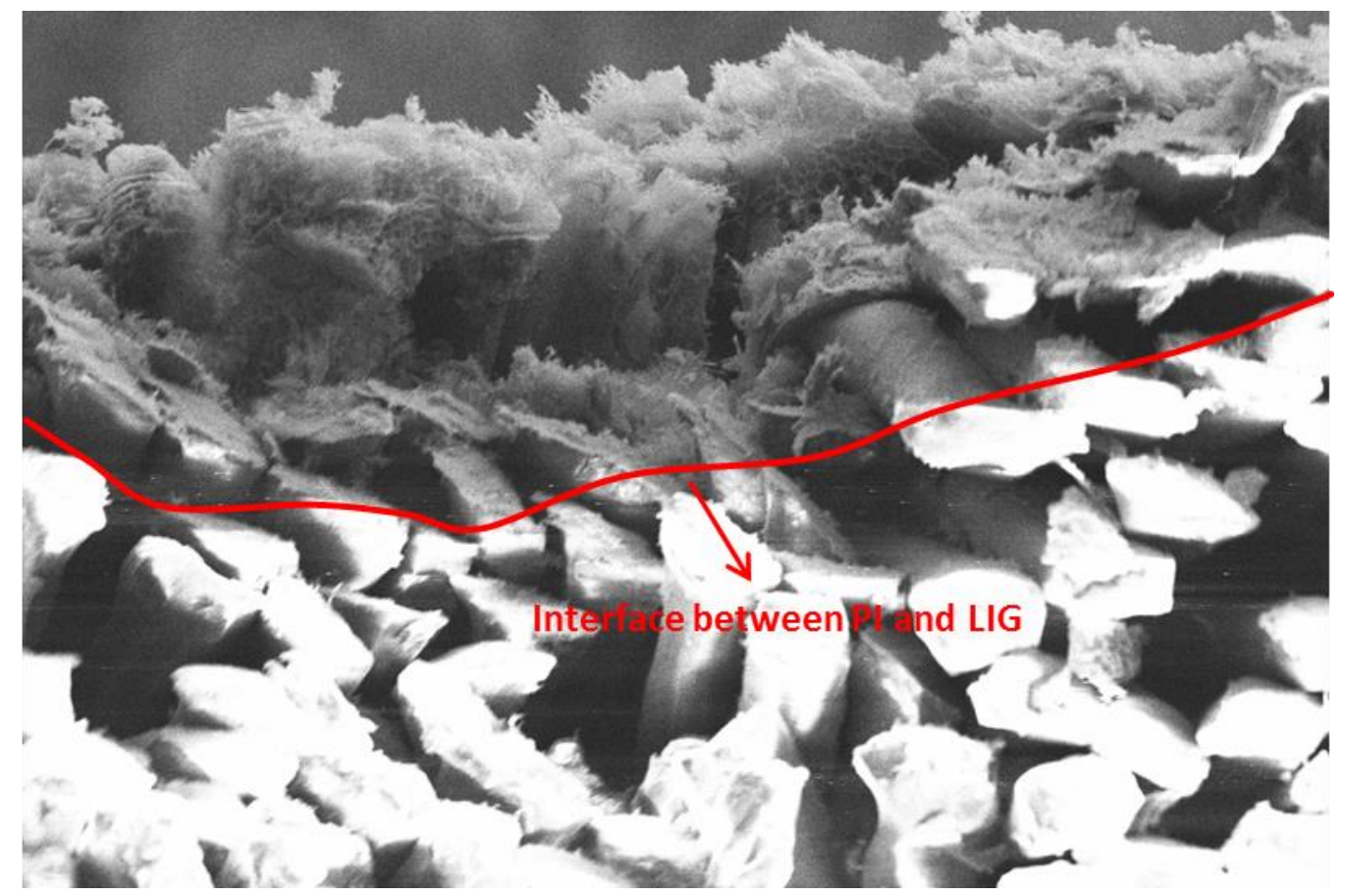

Figure S3. The cross-section SEM image of LIG grown on the PI fabric. 
XRD Spectrum of LIG on PI fabric with different laser fluence

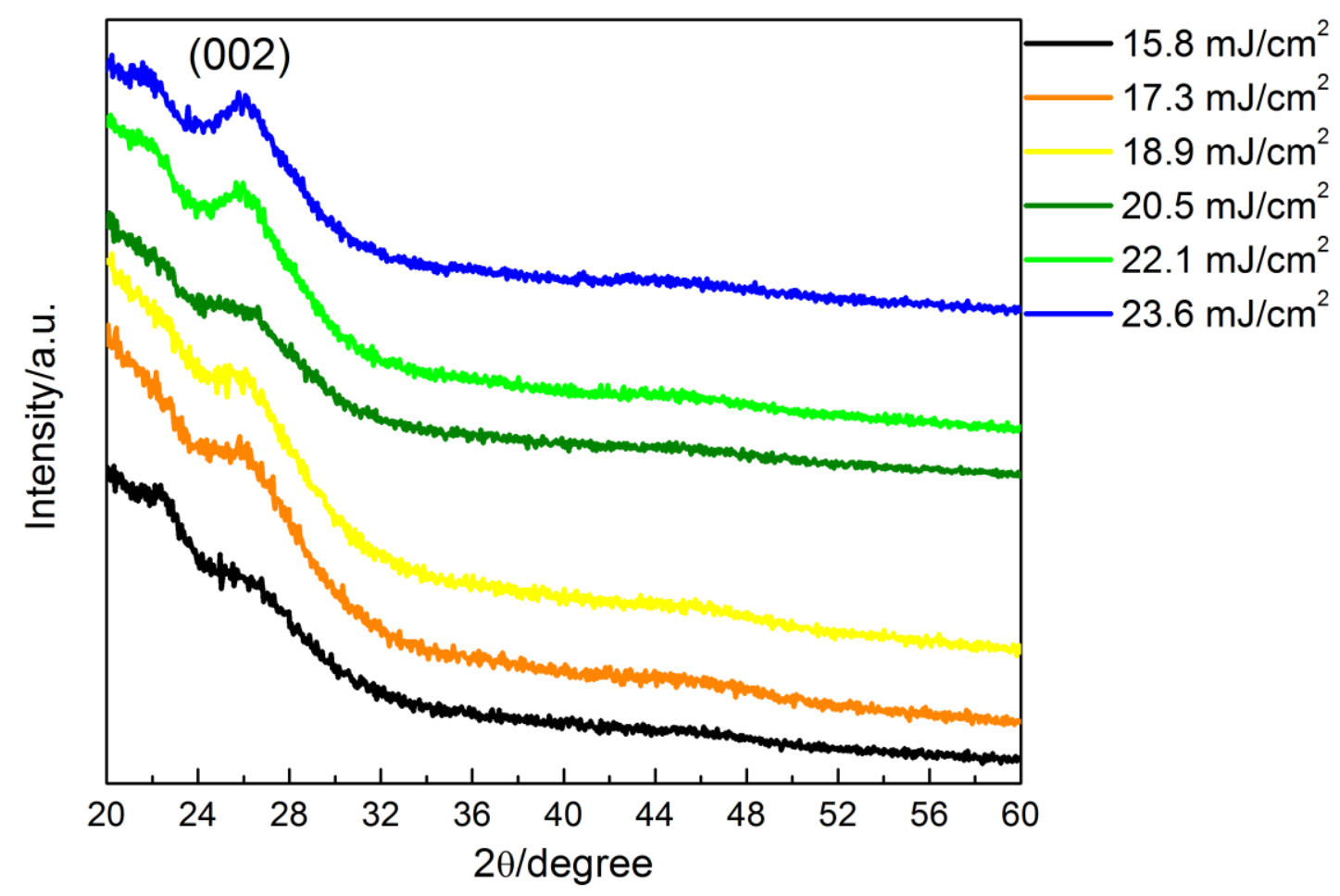

Figure S4. The XRD spectrum with different laser fluence. 
Resistance change of strain sensors with different laser fluence

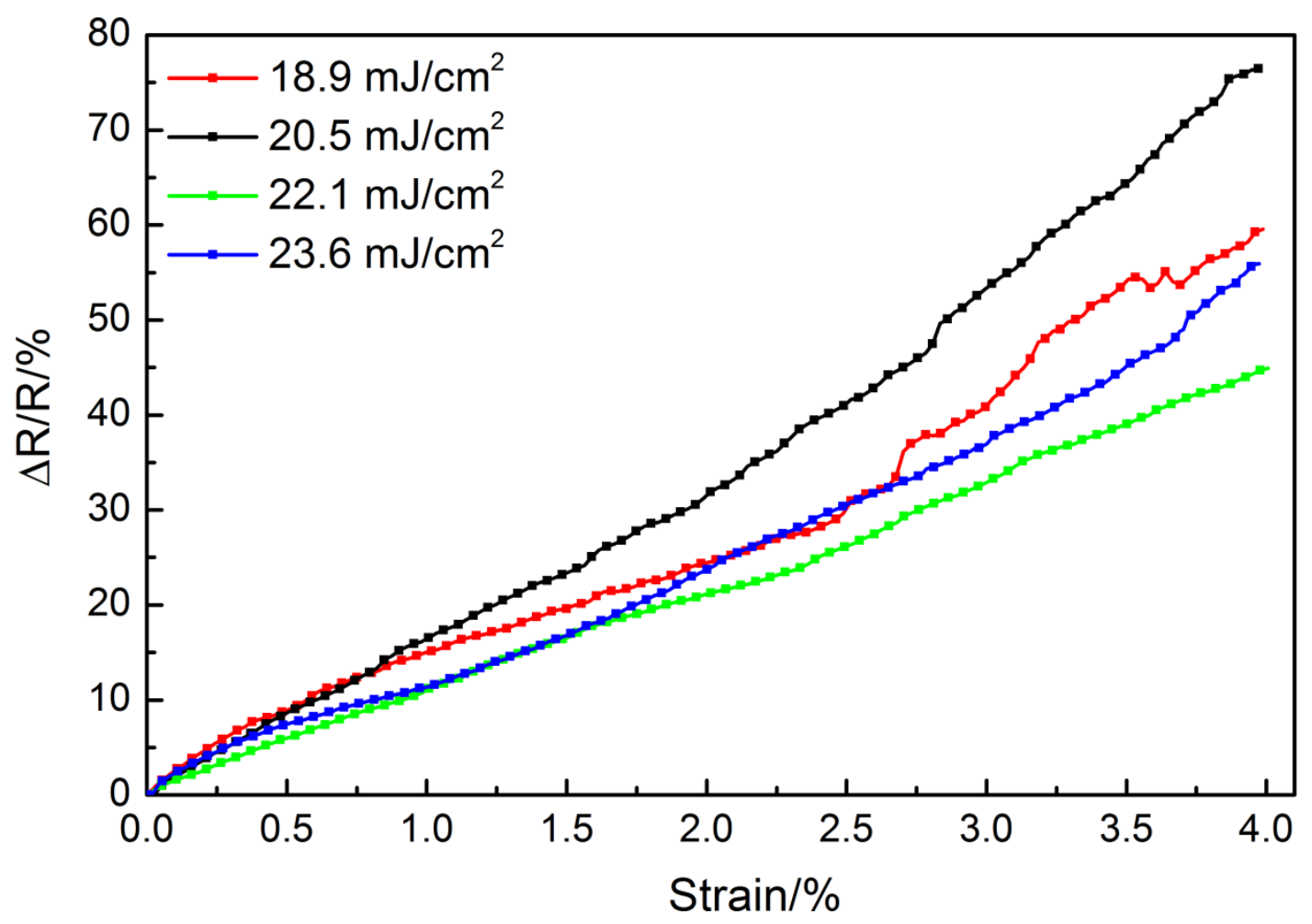

Figure S5. The curves of the resistance change versus strain of sensors synthesized with different laser fluence. 


\section{SEM image of nanoparticles on LIG}

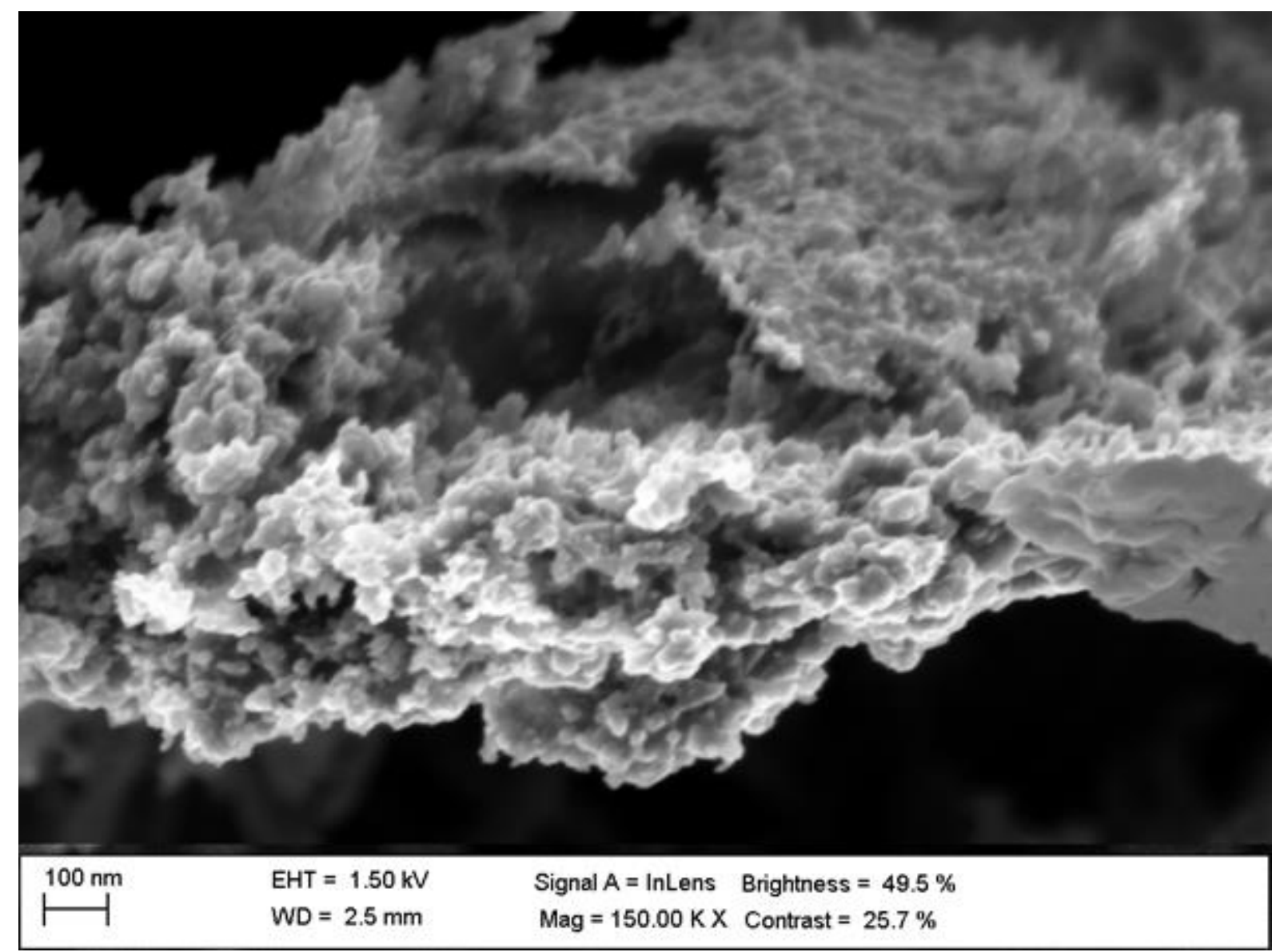

Figure S6. A SEM image of laser-scribed surface details showing the nanoparticles. 
SEM image of LIG/PI fabric in different directions under stretching

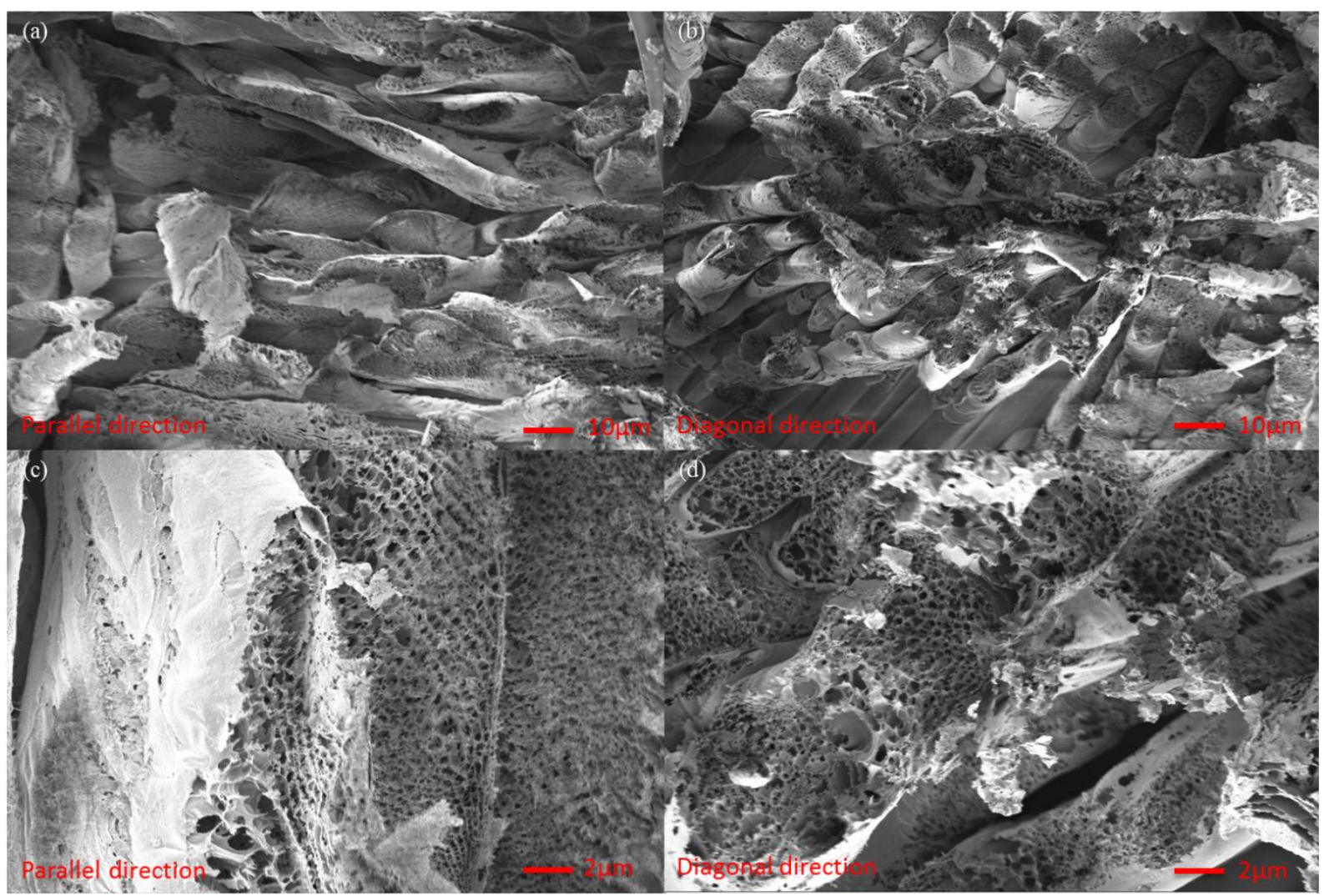

Figure S7. SEM images of the fiber surface morphology in two different directions under stretching: (a) X1000 magnification (parallel direction); (b) X1000 magnification(diagonal direction); (c) X5000 magnification (parallel direction); (d) X5000 magnification(diagonal direction). 
The resistance change versus strain plot within the different strain range
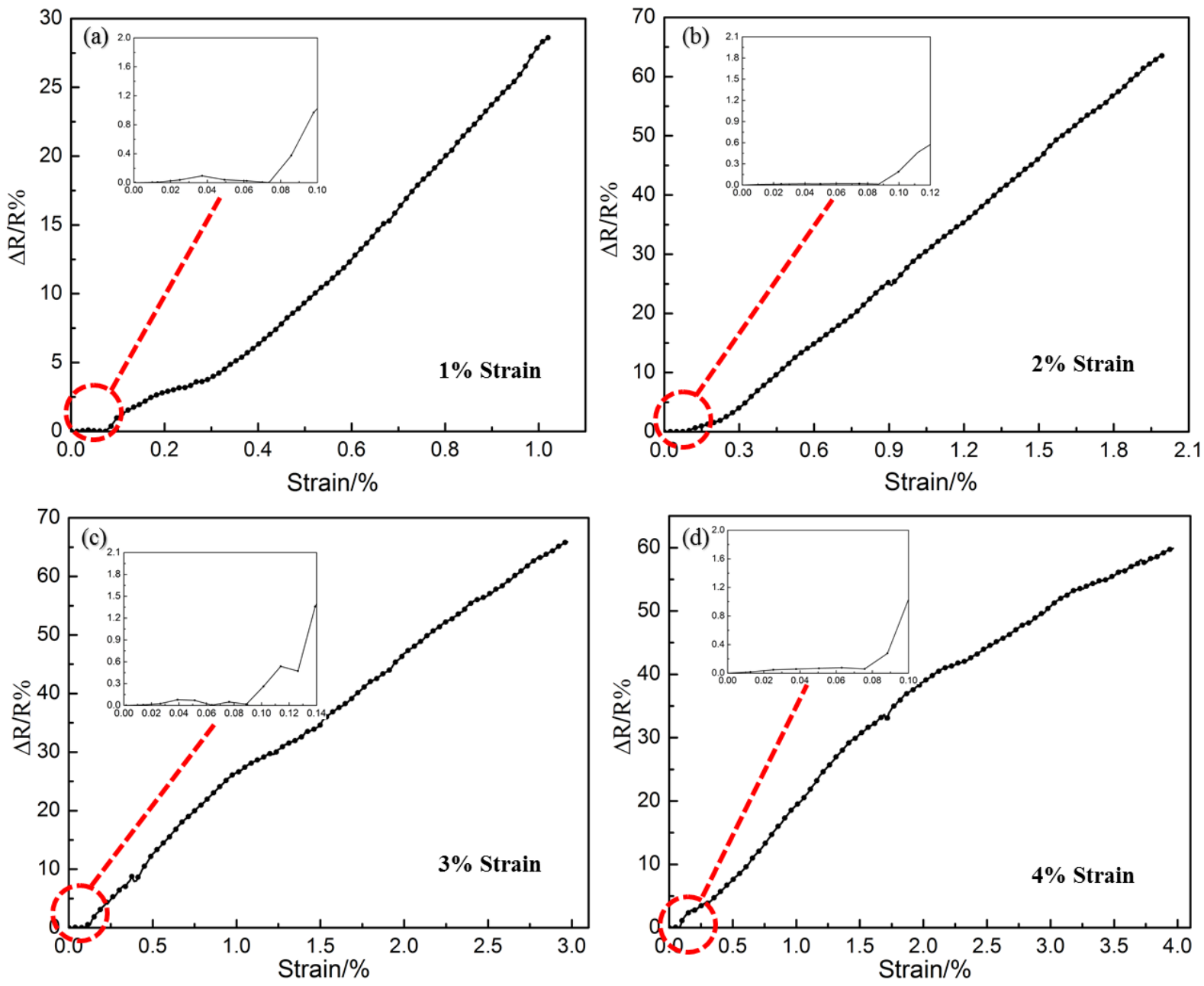

Figure S8. The resistance change versus strain plot within the different strain range: (a) $1 \%$; (b) $2 \%$; (c) $3 \%$; (d) $4 \%$ (Zoom-in figures presents plots within the strain range of $0.1 \%$ ). 
The fatigue test of PI fabric sensor

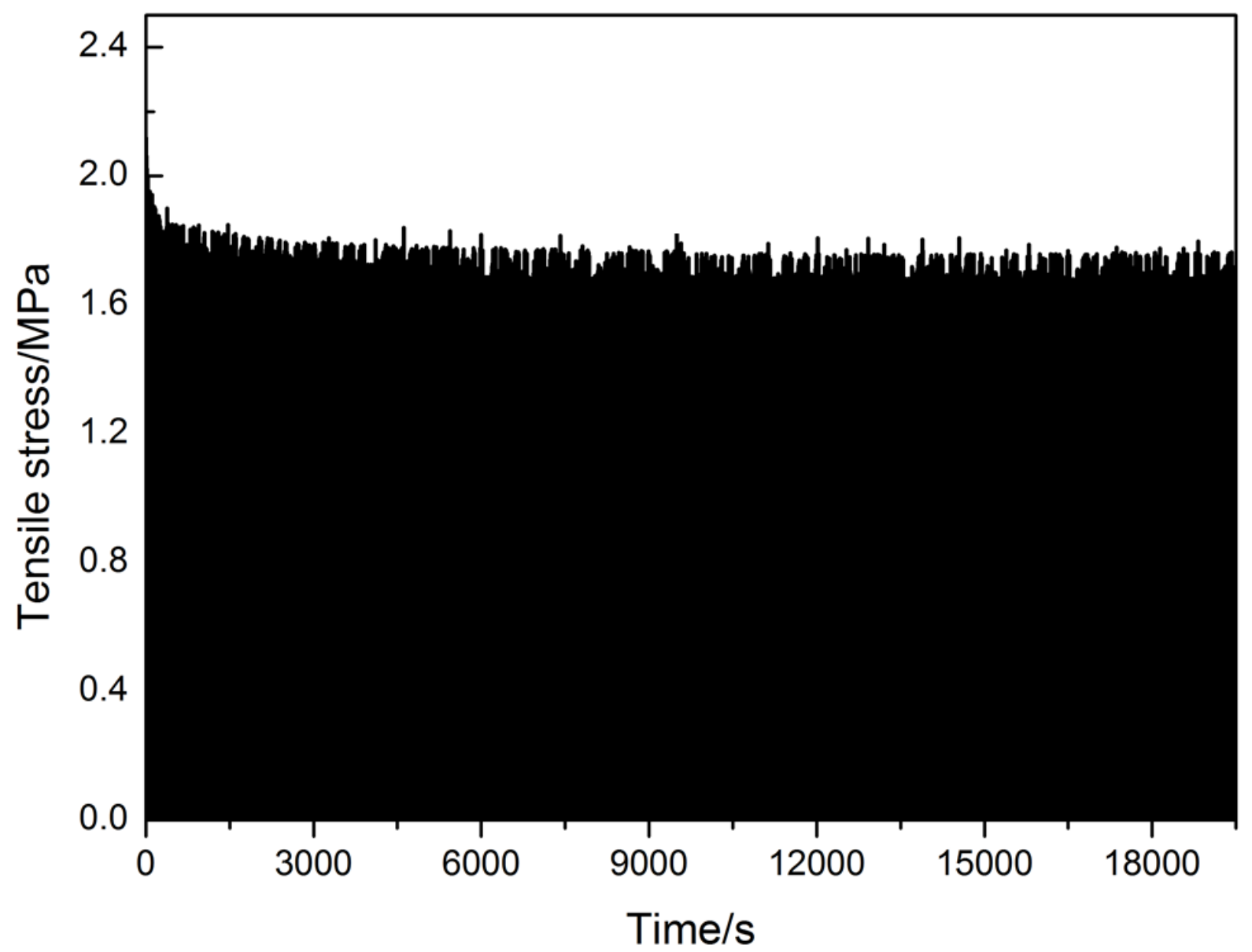

Figure S9. The fatigue test of PI fabric sensor after laser scribing under $4 \%$ strain for more than 6000 cycles. 
The sheet resistance of the LIG fabric sensor under washing cycles

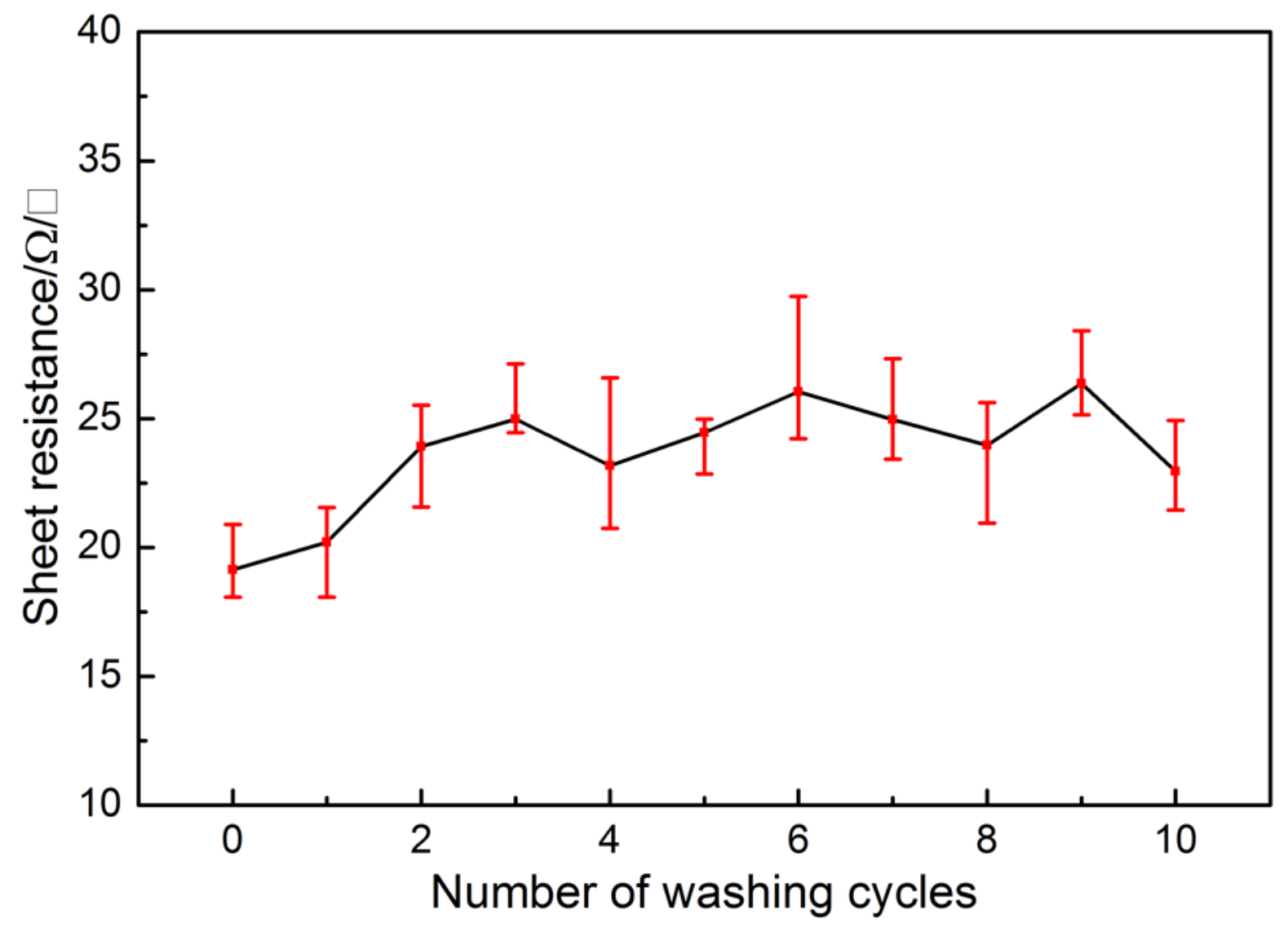

Figure S10. The sheet resistance of the LIG fabric sensor under 10 washing cycles. 
The specification table of aramid polyimide fiber woven fabric

Table S1| The specification of aramid polyimide fiber woven fabric

\begin{tabular}{|c|c|c|c|c|c|c|}
\hline $\begin{array}{l}\text { Product } \\
\text { Type }\end{array}$ & Pattern & Style & Width & Technics & Feature & weight \\
\hline $\begin{array}{l}\text { Aramid } \\
\text { Fabric }\end{array}$ & Printed & Twill & 58/60" & Woven & $\begin{array}{l}\text { Anti-Static, Bullet- } \\
\text { Proof, Chemical- } \\
\text { Resistant, Flame } \\
\text { Retardant, Heat- } \\
\text { Insulation }\end{array}$ & $190 \mathrm{~g} / \mathrm{m}^{2}$ \\
\hline
\end{tabular}


The comparison table of recent fabric strain sensors.

Table S2| The fabrication details and performance comparison of recent fabric strain sensors.

\begin{tabular}{|c|c|c|c|c|c|c|c|c|}
\hline $\begin{array}{l}\text { Sensing } \\
\text { mechanism }\end{array}$ & $\begin{array}{l}\text { Fabrication } \\
\text { method }\end{array}$ & Type & $\begin{array}{l}\text { Stretchable/Sensi } \\
\text { ng component }\end{array}$ & $\begin{array}{l}\text { Sensing } \\
\text { range }\end{array}$ & $\begin{array}{l}\text { Gauge } \\
\text { factor }\end{array}$ & Cyclic stability & Linearity & Ref \\
\hline Resistance & $\begin{array}{l}\text { In situ chemical } \\
\text { polymerization }\end{array}$ & Fiber & Polyester/ PEDOT & $\begin{array}{l}\text { Up to } \\
20 \%\end{array}$ & $\sim-0.76$ & $\begin{array}{c}1000 \\
\text { Cycles (at 10\% } \\
\text { strain) }\end{array}$ & Non-linear & 1 \\
\hline Resistance & Dip-coating & Fabric & Polyester/Ag & $6-50 \%$ & $\sim 5$ & $\begin{array}{c}1000 \text { cycles (at } \\
10 \% \text { strain) }\end{array}$ & Non-linear & 2 \\
\hline $\begin{array}{l}\text { Resistance } \\
\text { (current) }\end{array}$ & Spray coating & Fabric & $\begin{array}{c}\mathrm{PET} / \mathrm{ZnONW} \\
@ \mathrm{CNT} / \mathrm{rGO}\end{array}$ & $\begin{array}{l}\sim 6.2 \% \\
\text { Bending } \\
\text { strain }\end{array}$ & $\sim 7.6$ & $\begin{array}{c}200 \text { cycles (at } \\
\text { bending rates of } \\
0.2-1 \mathrm{~Hz} \text {. }\end{array}$ & Non-linear & 3 \\
\hline Resistance & Vacuum filtration & Fabric & Cotton/rGO & $\begin{array}{l}5-2.5 \mathrm{~cm} \\
\text { bending } \\
\text { radius }\end{array}$ & N/A & 400 cycles & Non-linear & 4 \\
\hline Resistance & Printing & Fabric & $\begin{array}{l}\text { Nylon 66/ } \\
\text { PEDOT:PSS }\end{array}$ & Up to $5 \%$ & $\sim-5$ & 25 cycles & Non-linear & 5 \\
\hline Resistance & $\mathrm{CPC}$ roller coating & Yarn & $\begin{array}{c}\text { Spandex/PU } \\
@ \text { CNT }\end{array}$ & $\begin{array}{l}\text { Up to } \\
30 \%\end{array}$ & $\sim 4.3$ & $\begin{array}{l}\text { Irreversible } \\
\text { signal } \\
\text { in } 15 \text { cycles }\end{array}$ & Non-linear & 6 \\
\hline Resistance & CVD & Yarn & None/CNT & $\begin{array}{l}\text { Up to } \\
3.6 \%\end{array}$ & $\sim 12$ & $\begin{array}{c}10 \text { cycles (at } 1 \% \\
\text { strain) }\end{array}$ & $\begin{array}{c}\text { High } \\
\text { linearity (at } \\
1 \% \text { strain) }\end{array}$ & 7 \\
\hline Resistance & $\begin{array}{l}\text { Melt-spinning } \\
\text { composite fiber }\end{array}$ & Fiber & PC MWCNT & Up to $6 \%$ & $\sim 16$ & N/A & Non-linear & 8 \\
\hline Resistance & Full-helical & Yarn & None/SWCNT & $\begin{array}{l}\text { Up to } \\
20 \%\end{array}$ & $\sim 0.1$ & $\begin{array}{l}\text { B90\% in } 1000 \\
\text { cycles }\end{array}$ & Non-linear & 9 \\
\hline Resistance & $\begin{array}{l}\text { Wet-spinning } \\
\text { composite fiber }\end{array}$ & Fiber & $\begin{array}{c}\text { SBS/ } \\
\text { AgNW@AgNP }\end{array}$ & $\begin{array}{l}\text { Up to } \\
100 \%\end{array}$ & $\sim 15$ & $\begin{array}{c}\sim 100 \% \\
\text { resistance } \\
\text { increase in } \\
1000 \text { cycles }\end{array}$ & Non-linear & 10 \\
\hline Resistance & CPC/LbL & Yarn & $\begin{array}{c}\mathrm{PU} / \\
\mathrm{CB} / \mathrm{CNC} / \mathrm{NR}\end{array}$ & Up to $5 \%$ & 38.9 & $\begin{array}{c}10000 \text { cycles (at } \\
1 \% \text { strain) }\end{array}$ & $\mathrm{N} / \mathrm{A}$ & 11 \\
\hline Resistance & $\begin{array}{l}\text { Hydrothermal } \\
\text { growth }\end{array}$ & Fiber & $\mathrm{PU} / \mathrm{ZnO}$ & $1.8-150 \%$ & $\begin{array}{c}\sim 15.2 \text { (at } \\
10 \% \\
\text { strain) } \\
\sim 4.1 \text { (at } \\
10-150 \%\end{array}$ & $\begin{array}{c}10000 \text { cycles } \\
\text { (at } 8.7-23.2 \% \\
\text { strain) }\end{array}$ & $\begin{array}{c}\text { High } \\
\text { linearity(at } \\
10-150 \% \\
\text { strain) }\end{array}$ & 12 \\
\hline Resistance & $\begin{array}{l}\text { CVD (Ni fabric } \\
\text { as template) }\end{array}$ & Fabric & PDMS/Graphene & Up to $3 \%$ & $\begin{array}{c}223 \text { (at } \\
3 \% \text { strain) }\end{array}$ & $\begin{array}{c}1000 \text { cycles (at } \\
3 \% \text { strain) }\end{array}$ & $\begin{array}{c}\text { High } \\
\text { linearity (at } \\
3 \% \text { strain) }\end{array}$ & 13 \\
\hline Resistance & $\begin{array}{l}\text { Laser-induced } \\
\text { graphene }\end{array}$ & Fabric & $\begin{array}{l}\text { Polyimide } \\
\text { fabric/LIG }\end{array}$ & Up to $4 \%$ & $\sim 27$ & $\begin{array}{c}1000 \text { cycles (at } \\
4 \% \text { strain) }\end{array}$ & $\begin{array}{l}\text { High } \\
\text { linearity }\end{array}$ & $\begin{array}{l}\text { Our } \\
\text { work }\end{array}$ \\
\hline
\end{tabular}




\section{Reference}

1. Eom, J.; Jaisutti, R.; Lee, H.; Lee, W.; Heo, J.-S.; Lee, J.-Y.; Park, S. K.; Kim, Y.-H., Highly sensitive textile strain sensors and wireless user-interface devices using all-polymeric conducting fibers. ACS applied materials \& interfaces 2017, 9 (11), 10190-10197.

2. Li, Y.; Li, Y.; Su, M.; Li, W.; Li, Y.; Li, H.; Qian, X.; Zhang, X.; Li, F.; Song, Y., Electronic textile by dyeing method for multiresolution physical kineses monitoring. Advanced Electronic Materials 2017, 3 (10), 1700253.

3. Lee, T.; Lee, W.; Kim, S. W.; Kim, J. J.; Kim, B. S., Flexible textile strain wireless sensor functionalized with hybrid carbon nanomaterials supported $\mathrm{ZnO}$ nanowires with controlled aspect ratio. Advanced Functional Materials 2016, 26 (34), 6206-6214.

4. Ren, J.; Wang, C.; Zhang, X.; Carey, T.; Chen, K.; Yin, Y.; Torrisi, F., Environmentally-friendly conductive cotton fabric as flexible strain sensor based on hot press reduced graphene oxide. Carbon 2017, 111, 622-630.

5. Calvert, P.; Duggal, D.; Patra, P.; Agrawal, A.; Sawhney, A., Conducting polymer and conducting composite strain sensors on textiles. Molecular Crystals and Liquid Crystals 2008, 484 (1), 291/[657]302/[668].

6. Zhang, R.; Deng, H.; Valenca, R.; Jin, J.; Fu, Q.; Bilotti, E.; Peijs, T., Carbon nanotube polymer coatings for textile yarns with good strain sensing capability. Sensors and Actuators A: Physical 2012, 179, 83-91.

7. Lekawa-Raus, A.; Koziol, K. K.; Windle, A. H., Piezoresistive effect in carbon nanotube fibers. ACS nano 2014, 8 (11), 11214-11224.

8. Bautista-Quijano, J. R.; Pötschke, P.; Brünig, H.; Heinrich, G., Strain sensing, electrical and mechanical properties of polycarbonate/multiwall carbon nanotube monofilament fibers fabricated by melt spinning. Polymer 2016, 82, 181-189.

9. Shang, Y.; He, X.; Li, Y.; Zhang, L.; Li, Z.; Ji, C.; Shi, E.; Li, P.; Zhu, K.; Peng, Q., Super - Stretchable Spring - Like Carbon Nanotube Ropes. Advanced Materials 2012, 24 (21), 2896-2900.

10. Lee, S.; Shin, S.; Lee, S.; Seo, J.; Lee, J.; Son, S.; Cho, H. J.; Algadi, H.; Al - Sayari, S.; Kim, D. E., Ag nanowire reinforced highly stretchable conductive fibers for wearable electronics. Advanced Functional Materials 2015, 25 (21), 3114-3121.

11. Wu, X.; Han, Y.; Zhang, X.; Lu, C., Highly sensitive, stretchable, and wash-durable strain sensor based on ultrathin conductive layer@ polyurethane yarn for tiny motion monitoring. ACS applied materials \& interfaces 2016, 8 (15), 9936-9945.

12. Liao, X.; Liao, Q.; Zhang, Z.; Yan, X.; Liang, Q.; Wang, Q.; Li, M.; Zhang, Y., A Highly Stretchable ZnO@ Fiber - Based Multifunctional Nanosensor for Strain/Temperature/UV Detection. Advanced Functional Materials 2016, 26 (18), 3074-3081.

13. Liu, X.; Tang, C.; Du, X.; Xiong, S.; Xi, S.; Liu, Y.; Shen, X.; Zheng, Q.; Wang, Z.; Wu, Y., A highly sensitive graphene woven fabric strain sensor for wearable wireless musical instruments. Materials Horizons 2017, 4 (3), 477-486. 